\title{
Risk for Invasive Cancers in Women With Breast Cancer In Situ: Results From a Population Not Covered by Organized Mammographic Screening
}

\author{
Nena Karavasiloglou ${ }^{1,2}$, Katarina L. Matthes ${ }^{1,2}$, Giulia Pestoni ${ }^{1,2}$, Manuela Limam ${ }^{1,2}$, \\ Dimitri Korol $^{2}$, Miriam Wanner ${ }^{1,2}$ and Sabine Rohrmann ${ }^{1,2 *}$ \\ ${ }^{1}$ Division of Chronic Disease Epidemiology; Epidemiology, Biostatistics and Prevention Institute (EBPl), University of Zurich, \\ Zurich, Switzerland, ${ }^{2}$ Cancer Registry of the Cantons of Zurich, Zug, Schaffhausen, and Schwyz, University Hospital Zurich, \\ Zurich, Switzerland
}

OPEN ACCESS

Edited by:

Hajo Zeeb,

Leibniz Institute for Prevention Research and Epidemiology (LG), Germany

Reviewed by: Abdelbaset Mohamed Elasbali, Al Jouf University, Saudi Arabia Jonas Czwikla,

University of Bremen, Germany

*Correspondence:

Sabine Rohrmann sabine.rohrmann@uzh.ch

Specialty section:

This article was submitted to Cancer Epidemiology and Prevention, a section of the journal

Frontiers in Oncology

Received: 15 September 2020 Accepted: 26 January 2021 Published: 18 March 2021

Citation:

Karavasiloglou N, Matthes KL

Pestoni G, Limam M, Korol D, Wanner M and Rohrmann S (2021) Risk for Invasive Cancers in Women With Breast Cancer In Situ: Results From a Population Not Covered by Organized Mammographic Screening.

Front. Oncol. 11:606747. doi: $10.3389 /$ fonc. 2021.606747
Background: Even though breast cancer in situ (BCIS) incidence has been increasing, the prognosis of BCIS patients has not been extensively investigated. According to the literature, women with $\mathrm{BCIS}$ have a higher risk of developing subsequent invasive breast cancer; conflicting information has been reported regarding their potential risk for a subsequent invasive non-breast cancer.

Methods: Data from 1,082 women, whose first-ever cancer diagnosis was primary BCIS between 2003 and 2015 and were living in the canton of Zurich, were used. Standardized incidence ratios (SIRs) were calculated to compare the risk of an invasive breast or nonbreast cancer among women with a primary BCIS with the corresponding risk of the adult female population. SIRs were calculated overall and by patient and tumor characteristics. To investigate potential risk factors (e.g., age at diagnosis, treatment) for a subsequent invasive breast or non-breast cancer we used Cox proportional hazards regression models.

Results: BCIS patients had 6.85 times [95\% confidence interval (Cl): 5.52-8.41] higher risk of being diagnosed with invasive breast cancer compared to the general population. They additionally faced 1.57 times (95\% Cl: 1.12-2.12) higher risk of an invasive nonbreast cancer. The SIRs were higher for women $<50$-years old for both invasive breast and non-breast cancer at BCIS diagnosis. Age $\geq 70$-years old at BCIS diagnosis was statistically significantly associated with a subsequent invasive nonbreast cancer diagnosis.

Conclusions: BCIS patients had a higher risk of being diagnosed with invasive breast and non-breast cancer compared to the general population. Age 70 years or older at BCIS diagnosis was the only risk factor statistically significantly associated with a subsequent invasive non-breast cancer. Our results support the increased risk for subsequent cancers in BCIS patients reported in the literature. Future studies should establish the risk factors for subsequent cancers, highlight the need for intensive monitoring in this population, and 
help distinguish BCIS patients who could benefit from systemic therapy to prevent distant cancers.

Keywords: breast cancer in situ, invasive cancer, risk, standardized incidence ratio, patients

\section{INTRODUCTION}

Breast cancer in situ (BCIS) is an intraepithelial lesion with malignant potential. Generally, BCIS is considered a nonobligatory precursor or a potential risk factor for invasive breast cancer, depending on the morphological subtype. Women with BCIS rarely report symptoms, and the majority of in situ tumors are detected through organized or opportunistic mammographic screening attendance (1).

Even though BCIS incidence has been increasing in the past decades (2-5) and it is estimated that BCIS cases account for up to $20 \%$ of the screen-diagnosed breast cancer cases (6), the clinical importance of BCIS remains unclear. Additionally, the prognosis of women diagnosed with BCIS has not been thoroughly investigated (6). Based on the existing literature, women with BCIS have a higher risk of developing a subsequent invasive breast cancer $(7-11)$, but the magnitude of the risk varies considerably (3.4 to 7.2 times higher than the risk in the comparison population). Most of the aforementioned studies have been conducted in regions with established organized mammographic screening programs or where organized mammographic screening programs were established during the study period $(9,12)$. Investigating the risk for subsequent breast cancer in women with BCIS in regions where only opportunistic screening exists will improve our understanding of the progression of the disease since fewer women are using mammograms in these regions $(13,14)$. In the absence of organized screening, a smaller, selective number of women will choose to undergo mammography for various reasons (e.g., health-consciousness, family history of breast cancer), meaning that some breast cancer in situ cases might not be diagnosed. Reports from Switzerland indicate that a lower proportion of women undergoes mammography in the German-speaking regions, where mainly opportunistic screening exists, compared to the French-speaking regions, where organized screening programs have been established (34.9 vs. $77.8 \%$ of $50-69$ yearolds reported a mammogram in the past 2 years, respectively) $(13,14)$.

Furthermore, the findings regarding the risk of BCIS patients for subsequent invasive cancer in sites other than the breast are contradicting. A previous Swiss study including data from the Canton of Vaud did not report an increased risk for invasive cancer in sites other than the breast (7) compared to the comparison population, while a study conducted in the Netherlands suggested an increased risk in BCIS patients (10).

We aimed to investigate the risk for a subsequent invasive cancer (breast and non-breast) in BCIS patients in the Canton of Zurich, where no organized mammographic screening program exists. To the best of our knowledge, this is the first study to report on the risk for subsequent invasive cancer after BCIS diagnosis in the German-speaking region of Switzerland.

\section{METHODS}

\section{Population}

We used data from women whose first-ever cancer diagnosis was primary BCIS [D05.0-D05.9; International Statistical Classification of Diseases and Related Health Problems, 10th revision (ICD-10)] and occurred between 2003 and 2015 (hereafter referred to as primary BCIS). The data were obtained from the Cancer Registry of the Cantons of Zurich, Zug, Schaffhausen, and Schwyz. A recent publication demonstrated that the data quality from the Cancer Registry was acceptable based on four widely used data quality indicators (comparability, validity, timeliness, and completeness) (15). The Cancer Registry started recording cases for the Cantons of Zug, Schaffhausen, and Schwyz later (2011, 2020, and 2020, respectively), so the present analyses only focus on data from the Canton of Zurich. To be included in the study, patients had to live in the canton of Zurich at the time of diagnosis, even if they were treated in another canton. BCIS with morphological codes [according to the International Classification of Diseases for Oncology, 3rd Edition (ICD-O-3)] 8201/2, 8230/2, 8500/2, $8501 / 2,8503 / 2,8504 / 2$, and $8507 / 2$ were categorized as ductal carcinoma in situ. Cases with ICD-O-3 morphological code $8520 / 2$ were categorized as lobular carcinoma in situ, and cancers with ICD-O-3 morphological code 8522/2 as unspecified cancers in situ. We excluded from our analyses patients diagnosed with a recorded cancer before the BCIS, patients diagnosed with Paget disease of the breast, and patients for whom invasive breast cancer was diagnosed simultaneously with the BCIS. Patients with unknown laterality of BCIS and those diagnosed with bilateral BCIS were also excluded from our analyses.

Our outcomes of interest were invasive breast cancer diagnosis (C50, ICD-10) and invasive non-breast cancer diagnosis (i.e., all sites except breast and non-melanoma skin cancer combined; C00-C96 and D45-D47, excluding C50, ICD10). The latter tumors (D45-D47, ICD-10) were included in our analyses as invasive non-breast tumors due to their malignant behavior according to ICD-O-3. Thus, patients diagnosed with a second primary in situ cancer were excluded from our analyses $(n=39)$. When looking at the risk of invasive breast cancer, patients treated with double mastectomy $(n=3)$ for their primary BCIS were excluded from the analyses given their very low risk for subsequent invasive breast cancer. However, these patients were retained when looking at the subsequent risk of invasive non-breast cancer. 
Time at risk for an invasive cancer was assumed to begin 3 months after primary BCIS and lasted until the date of an invasive cancer diagnosis, date of emigration, date of death or end of follow-up (31st December 2016), whichever came first. Thus, patients with less than 3 months of follow-up after their primary BCIS diagnosis were excluded from our analysis. We obtained patients' vital status from the Citizen Services Departments of the Canton of Zurich. Our final study population included 1,082 patients with primary BCIS diagnosis (1,079 patients when investigating invasive breast cancer risk).

Given the different treatment schemes for BCIS patients, we only focused on the first available treatment after BCIS diagnosis. Treatment options were grouped into four categories as follows: breast-conserving surgery (including quadrantectomy and tumorectomy, with or without lymph node dissection), mastectomy or surgery not otherwise specified (NOS), radiotherapy, hormonal therapy, or other therapies, and unknown.

\section{Statistical Analyses}

Standardized incidence ratios (SIRs) were calculated to compare the risk of invasive breast or non-breast cancer among women with primary BCIS with the corresponding risk of primary invasive breast or primary invasive non-breast tumor in the adult female population of the canton of Zurich. The observed number of invasive breast or non-breast cancers was counted among the index cohort by year and age group at diagnosis $(<50,50-59,60-69, \geq 70$ years old). The expected number of invasive cancers was estimated among the comparison cohort by multiplying the person-time of follow-up with the corresponding age- and period-specific incidence rates. Ninety-five percent of confidence intervals (CIs) for the SIRs were estimated by assuming Poisson distribution for the observed cases and using Wald's normal-approximation [popEpi package in $\mathrm{R}(16)$ ]. Stratified analyses by morphological subtype, laterality and treatment of primary BCIS, year of primary BCIS diagnosis, age group at primary BCIS diagnosis, and calendar period of primary BCIS diagnosis were performed. Given the small number of patients with unspecified BCIS $(n=7)$, the separate results for this morphological subtype are not shown.

We used univariate Cox proportional hazards regression models to investigate which factors are associated with the risk of being subsequently diagnosed with invasive breast or nonbreast cancer [survival package in R (17)]. Entry time started 3 months after a participant's BCIS diagnosis and exit time was defined as the date of diagnosis of subsequent cancer. Participants who were not diagnosed with a subsequent cancer were censored on the date of loss to follow-up, end of follow-up (31st December 2016), or death, whichever came first. Age at BCIS diagnosis, treatment, morphological subtype and laterality of the initial BCIS tumor, as well as sociodemographic factors (nationality and marital status) were investigated as potential risk factors for subsequent invasive cancer diagnosis. Analyses were conducted separately for the risk of invasive breast and non-breast cancer. Results were presented as hazard ratios (HRs) and $95 \%$ CIs.

We conducted sensitivity analyses, retaining only women who had at least 6 months of follow-up. All analyses were performed in $\mathrm{R}$ (version 3.5.0, R Foundation for Statistical Computing, Vienna, Austria) and significance levels were set at alpha $=0.05$.

\section{RESULTS}

Description of the study population and baseline characteristics are shown in Table 1. The most frequent invasive cancer diagnoses in BCIS patients were invasive breast, gynecological, or colorectal cancers. Of BCIS patients, those subsequently diagnosed with invasive non-breast cancer (i.e., all sites except breast and non-melanoma skin cancer combined) were older at the time of their initial BCIS diagnosis than both patients who

TABLE 1 | Characteristics of study participants based on whether or not they received a second primary cancer diagnosis during the study period. ${ }^{1}$

\begin{tabular}{|c|c|c|c|}
\hline & \multirow{2}{*}{$\begin{array}{l}\text { No second } \\
\text { primary } \\
\text { diagnosis } \\
(n=950)\end{array}$} & \multicolumn{2}{|c|}{$\begin{array}{l}\text { Second primary diagnosis } \\
\qquad(n=132)\end{array}$} \\
\hline & & $\begin{array}{l}\text { Invasive } \\
\text { breast } \\
\text { cancer } \\
(n=91)\end{array}$ & $\begin{array}{l}\text { Invasive } \\
\text { non- } \\
\text { breast } \\
\text { cancer } \\
(\mathrm{n}=41)\end{array}$ \\
\hline $\begin{array}{l}\text { Age at diagnosis of primary BCIS } \\
\text { (years, mean } \pm \text { SD) } \\
\text { Time period of primary BCIS } \\
\text { diagnosis, } n(\%)\end{array}$ & $57.2 \pm 11.2$ & $56.8 \pm 11.8$ & $63.5 \pm 13.1$ \\
\hline 2003-2009 & $433(45.6)$ & $59(64.8)$ & $29(70.7)$ \\
\hline 2010-2015 & $517(54.4)$ & $32(35.2)$ & $12(29.3)$ \\
\hline \multicolumn{4}{|l|}{ Treatment of primary BCIS, n (\%) } \\
\hline Breast-conserving surgery & $639(67.3)$ & $54(59.3)$ & $22(53.7)$ \\
\hline Mastectomy or Surgery, NOS ${ }^{2}$ & $188(19.8)$ & $17(18.7)$ & $9(22.0)$ \\
\hline $\begin{array}{l}\text { Radiotherapy, Hormonal therapy } \\
\text { or Other therapy }\end{array}$ & $82(8.6)$ & $12(13.2)$ & $7(17.1)$ \\
\hline Unknown & $41(4.3)$ & $8(8.8)$ & $3(7.3)$ \\
\hline \multicolumn{4}{|l|}{$\begin{array}{l}\text { Morphological subtype of primary } \\
\text { BCIS, } n(\%)\end{array}$} \\
\hline Ductal & $863(90.8)$ & $80(87.9)$ & $35(85.4)$ \\
\hline Lobular & $81(8.5)$ & $11(12.1)$ & $5(12.2)$ \\
\hline Unspecified & $6(0.6)$ & - & $1(2.4)$ \\
\hline \multicolumn{4}{|l|}{ Laterality of primary BCIS, n (\%) } \\
\hline Right & $440(46.3)$ & $49(53.8)$ & $19(46.3)$ \\
\hline Left & $510(53.7)$ & $42(46.2)$ & $22(53.7)$ \\
\hline \multicolumn{4}{|l|}{ Marital status, n (\%) } \\
\hline $\begin{array}{l}\text { Never married, widowed, } \\
\text { divorced, or Separated }\end{array}$ & $254(26.7)$ & $22(24.2)$ & $17(41.5)$ \\
\hline Married or living with partner & $451(47.5)$ & $54(59.3)$ & $16(39.0)$ \\
\hline Unknown & $245(25.8)$ & $15(16.5)$ & $8(19.5)$ \\
\hline \multicolumn{4}{|l|}{ Nationality, n (\%) } \\
\hline Swiss & $684(72.0)$ & $71(78.0)$ & $33(80.5)$ \\
\hline Non-Swiss & $135(14.2)$ & $14(15.4)$ & $3(7.3)$ \\
\hline Unknown & $131(13.8)$ & $6(6.6)$ & $5(12.2)$ \\
\hline
\end{tabular}

${ }^{1} B C / S$, breast cancer in situ; NOS, not otherwise specified; SD, standard deviation. ${ }^{2}$ Excluding double mastectomy. 
were not subsequently diagnosed with invasive cancer and those subsequently diagnosed with invasive breast cancer.

The overall age-standardized incidence rate (ASR) for primary invasive breast cancer in the adult female population of the Canton of Zurich during our study period (2003-2016) was 104.2 per 100,000 person-years. The corresponding ASR for primary invasive non-breast cancer (i.e., all sites except breast and non-melanoma skin cancer combined) was 195.7 per 100,000 person-years.

The results of the risk for subsequent invasive breast cancer after BCIS diagnosis compared to the risk of the general adult female population of Zurich for primary invasive breast cancer are shown in Table 2. After 6,362 person-years, BCIS patients had 6.85 (95\% CI: 5.52-8.41) times higher risk of receiving an invasive breast cancer diagnosis compared to the general adult female population of Zurich. Women diagnosed with BCIS before the age of 50 had higher SIR (21.74, 95\% CI: 14.6932.18) for invasive breast cancer. Higher SIR was also observed for women diagnosed with BCIS in their right breast $(7.96,95 \%$ CI: 6.01-10.53); however, the CIs of the SIRs of women diagnosed with BCIS in their right breast, compared to those diagnosed with BCIS in their left breast were overlapping. The SIRs did not vary considerably by morphological subtype or treatment of the initial BCIS (Table 2).

Regarding the risk for invasive non-breast cancer, after 6,371 person-years, BCIS patients had 1.57 (95\% CI: 1.12-2.12) times higher risk of being diagnosed with invasive non-breast cancer, compared to the risk of primary invasive non-breast cancer in the general adult female population of Zurich. Women diagnosed with BCIS before the age of 50 had higher SIR (4.02, 95\% CI: 1.81-8.96) for invasive non-breast cancer; however, the
CI of the SIR were overlapping with those of other age groups. The risk did not vary considerably by morphological subtype, laterality, treatment of the initial BCIS, or time-period of the BCIS diagnosis (Table 2).

The association between sociodemographic and tumor characteristics and risk for a subsequent invasive (breast or non-breast) cancer is shown in Table 3. In the univariate Cox models, older age at BCIS diagnosis ( $\geq 70$ years old) was statistically significantly associated with increased risk for a subsequent invasive non-breast cancer (HR: 3.34, 95\% CI: 1.43-7.82), but not for subsequent invasive breast cancer (HR: 1.21, 95\% CI: 0.65-2.27). No associations between morphological subtype, treatment, laterality, time-period of diagnosis (20032009 vs. 2010-2015), or age at diagnosis of the primary BCIS diagnosis were seen with either invasive breast or non-breast cancer risk. Sensitivity analyses excluding women with less than 6 months of follow-up did not alter our results (data not shown).

\section{DISCUSSION}

In our study, we observed an increased risk of a subsequent invasive breast or non-breast cancer diagnosis in BCIS patients compared to the general adult female population of Zurich. Older age at primary BCIS diagnosis was associated with the risk of a subsequent invasive non-breast cancer, but not with subsequent invasive breast cancer.

Overall, our findings suggest that BCIS patients have approximately 6.9 times higher risk for invasive breast cancer compared to the general adult female population of Zurich. These results are in line with previous Swiss and international

TABLE 2 | Standardized incidence ratios (SIRs) for an invasive cancer diagnosis (breast or non-breast) after diagnosis of primary breast cancer in situ (BCIS) and its 95\% confidence interval $(\mathrm{Cl})$, overall and by patient and tumor characteristics. ${ }^{1}$

\begin{tabular}{|c|c|c|c|c|}
\hline & \multicolumn{2}{|c|}{ Invasive breast cancer } & \multicolumn{2}{|c|}{ Invasive non-breast cancer } \\
\hline & O/E & SIR (95\% CI) & O/E & SIR (95\% CI) \\
\hline Overall & $91 / 13.28$ & $6.85(5.52-8.41)$ & $41 / 26.18$ & $1.57(1.12-2.12)$ \\
\hline \multicolumn{5}{|l|}{ Morphological subtype of primary BCIS } \\
\hline Ductal & $80 / 11.80$ & $6.78(5.45-8.44)$ & $35 / 23.44$ & $1.49(1.07-2.08)$ \\
\hline Lobular & $11 / 1.33$ & $8.26(4.58-14.92)$ & $5 / 2.49^{\star}$ & $2.01(0.84-4.83)$ \\
\hline \multicolumn{5}{|l|}{ Treatment of primary BCIS } \\
\hline Breast-conserving surgery & $54 / 7.96$ & $6.79(5.20-8.86)$ & $22 / 15.53$ & $1.42(0.93-2.15)$ \\
\hline Mastectomy or Surgery, NOS & $17 / 2.85$ & $5.96(3.71-9.59)$ & $9 / 5.88$ & $1.53(0.80-2.94)$ \\
\hline Radiotherapy, hormonal therapy, or other therapies & $12 / 1.87$ & $6.40(3.63-11.27)$ & $7 / 3.58$ & $1.96(0.93-4.11)$ \\
\hline Unknown & $8 / 0.60$ & $13.36(6.68-26.71)$ & $3 / 1.19$ & $2.51(0.81-7.79)$ \\
\hline \multicolumn{5}{|l|}{ Laterality of primary BCIS } \\
\hline Right & $49 / 6.16$ & $7.96(6.01-10.53)$ & $19 / 12.19$ & $1.56(0.99-2.44)$ \\
\hline Left & $42 / 7.12$ & $5.90(4.36-7.98)$ & 22/13.99 & $1.57(1.04-2.39)$ \\
\hline \multicolumn{5}{|l|}{ Age at diagnosis of primary BCIS } \\
\hline$<50$ years & $25 / 1.15$ & $21.74(14.69-32.18)$ & $6 / 1.49$ & $4.02(1.81-8.96)$ \\
\hline $50-59$ years & $28 / 4.60$ & $6.09(4.20-8.82)$ & $9 / 7.08$ & $1.27(0.66-2.44)$ \\
\hline $60-69$ years & $23 / 4.48$ & $5.13(3.41-7.72)$ & $13 / 8.47$ & $1.53(0.89-2.64)$ \\
\hline$\geq 70$ years & 15/3.05 & $4.92(2.97-8.16)$ & $13 / 9.14$ & $1.42(0.83-2.45)$ \\
\hline \multicolumn{5}{|l|}{ Time-period of diagnosis of primary BCIS } \\
\hline 2003-2009 & $59 / 9.24$ & $6.39(4.95-8.24)$ & 29/18.14 & $1.60(1.11-2.30)$ \\
\hline 2010-2015 & $32 / 4.04$ & $7.92(5.60-11.20)$ & $12 / 8.04$ & $1.49(0.85-2.63)$ \\
\hline
\end{tabular}

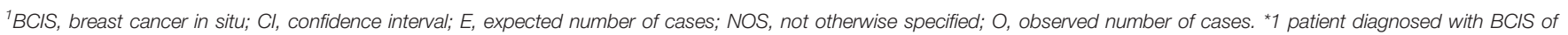
unspecified morphological subtype. Given the low count in the Unspecified subtype results are not shown separately for it. 
TABLE 3 | Cox regression (univariate analyses) of subsequent invasive cancer diagnosis by potential risk factors following in situ breast cancer diagnosis. ${ }^{1}$

\begin{tabular}{|c|c|c|}
\hline & $\begin{array}{l}\text { Invasive breast } \\
\text { cancer }\end{array}$ & $\begin{array}{c}\text { Invasive non-breast } \\
\text { cancer }\end{array}$ \\
\hline & HR $(95 \% \mathrm{Cl})$ & HR $(95 \% \mathrm{Cl})$ \\
\hline \multicolumn{3}{|l|}{ Tumor characteristics } \\
\hline \multicolumn{3}{|l|}{ Morphological subtype of primary } \\
\hline \multicolumn{3}{|l|}{$\mathrm{BCIS}$} \\
\hline Ductal & Ref. & Ref. \\
\hline Lobular & $1.14(0.61-2.15)$ & $1.14(0.44-2.90)$ \\
\hline \multicolumn{3}{|l|}{ Treatment of primary BCIS } \\
\hline Breast-conserving surgery & Ref. & Ref. \\
\hline Mastectomy or surgery, NOS & $0.88(0.51-1.53)$ & $1.07(0.49-2.34)$ \\
\hline $\begin{array}{l}\text { Radiotherapy, hormonal therapy, or } \\
\text { other therapies }\end{array}$ & $1.05(0.56-1.98)$ & $1.31(0.55-3.10)$ \\
\hline Unknown & $1.97(0.94-4.14)$ & $1.74(0.52-5.80)$ \\
\hline \multicolumn{3}{|l|}{ Laterality of primary BCIS } \\
\hline Right & Ref. & Ref. \\
\hline Left & $0.75(0.49-1.13)$ & $1.02(0.55-1.88)$ \\
\hline \multicolumn{3}{|l|}{$\begin{array}{l}\text { Time-period of diagnosis of primary } \\
\text { BCIS }\end{array}$} \\
\hline 2003-2009 & Ref. & Ref. \\
\hline 2010-2015 & $1.02(0.64-1.62)$ & $0.99(0.46-2.11)$ \\
\hline \multicolumn{3}{|l|}{ Patient characteristics } \\
\hline \multicolumn{3}{|l|}{ Age at diagnosis of primary $\mathrm{BCIS}$} \\
\hline$<50$ years & $1.21(0.71-2.08)$ & $0.89(0.32-2.51)$ \\
\hline 50-59 years & Ref. & Ref. \\
\hline 60-69 years & $1.21(0.70-2.10)$ & $2.16(0.92-5.04)$ \\
\hline$\geq 70$ years & $1.21(0.65-2.27)$ & $3.34(1.43-7.82)$ \\
\hline \multicolumn{3}{|l|}{ Marital status } \\
\hline Married or living with partner & Ref. & Ref. \\
\hline $\begin{array}{l}\text { Never married, widowed, divorced, } \\
\text { or separated }\end{array}$ & $0.71(0.43-1.16)$ & $1.66(0.84-3.28)$ \\
\hline Unknown & $0.51(0.29-0.90)$ & $0.86(0.37-2.00)$ \\
\hline \multicolumn{3}{|l|}{ Nationality } \\
\hline Swiss & Ref. & Ref. \\
\hline Non-Swiss & $1.22(0.69-2.17)$ & $0.60(0.18-1.95)$ \\
\hline Unknown & $0.37(0.16-0.86)$ & $0.63(0.25-1.62)$ \\
\hline
\end{tabular}

${ }^{1} \mathrm{BCIS}$, breast cancer in situ; $\mathrm{Cl}$, confidence interval; HR, hazard ratio; NOS, not otherwise specified. Given the low count of patients diagnosed with BCIS of unspecified morphological subtype, results are not shown separately for that subtype.

studies suggesting an increased risk of a subsequent invasive breast cancer in BCIS patients $(7-11,18)$. In the literature, the risk for subsequent invasive breast cancer in BCIS patients ranges from 3.4 to 7.2 times higher compared to the risk in the comparison population. Our estimates were at the higher end of those reported in the literature, potentially reflecting the lack of organized screening programs in our region (i.e., we are identifying less in situ tumors compared to regions with organized screening programs). Some studies looking at the SIRs in regions where organized mammographic screening programs have been implemented suggest that the SIRs are high before and during the implementation of the screening programs, but start decreasing long after implementation (10, 12). It is assumed that with screening in place, all BCIS will be detected, not only those in specific women who undergo elective screening, and treated early, before the detection of invasive components. Based in a previous Swiss study, fewer women reported having had a mammography in the past 2 years in the German-speaking regions compared to the French-speaking regions (34.9 vs. $77.8 \%$ of 50-69 year olds, respectively) (13).
Another potential explanation for our findings is that the in situ lesions in our study population had progressed to higher grade before detection. Our previous findings suggest that onethird of the breast cancer in situ lesions in the Canton of Zurich were of high grade (2), indicating that in situ tumors in our study population progressed to higher grade before detection. Previous studies suggest that BCIS gradually evolves from low grade, welldifferentiated lesions to high grade, poorly differentiated lesions by acquiring genetic mutations (19).

Regarding the risk of invasive non-breast cancer after BCIS diagnosis, the findings in the literature are contradicting. While a Swiss study reported no increased risk for invasive non-breast cancer in BCIS patients (7), a study conducted in the Netherlands suggested a 1.4 times increased risk (10). Our findings are in line with the latter study, adding to the literature suggesting that BCIS patients are at higher risk for both invasive breast and invasive non-breast cancer after their initial BCIS diagnosis. This increased risk could be attributed to increased surveillance in BCIS patients after their cancer diagnosis, compared to the general population, or could be an indication of genetic or lifestyle factors that are associated with increased risk for both BCIS and invasive cancers.

Older age at BCIS diagnosis ( $\geq 70$ years old) was statistically significantly associated with a subsequent invasive non-breast cancer, but not with a subsequent invasive breast cancer diagnosis in the univariate Cox models. The lack of statistically significant associations with the majority of the factors investigated in this study could suggest that basic primary tumor or patient characteristics might not be strongly associated with subsequent invasive cancers after an initial BCIS diagnosis. Some studies in the literature also failed to detect such associations. However, larger studies have reported associations of younger age at diagnosis of the initial BCIS, race, or family history for breast cancer with subsequent cancer risk $(9,12,20)$. The direction of our results regarding younger age at diagnosis for invasive breast cancer are consistent with those reported in the literature. Another possible explanation is that the classification we used for some factors (e.g., primary BCIS treatment grouped in four categories) did not allow for possible associations to be detected as statistically significant. This classification was used to accommodate the low number of cases in the Canton of Zurich. Thus, future projects should aim to include larger BCIS populations to be able to detect potential associations between primary tumor characteristics, as well as patient characteristics and subsequent invasive cancer diagnoses.

Our study had several strengths. Given the high registry coverage in the Canton of Zurich, we are confident that we capture almost all incident cancer cases in the canton (15). Additionally, medical and treatment information, as well as patient and tumor characteristics were available for a high proportion of our study population, allowing us to stratify the SIRs for these factors while also assessing them as potential risk factors for subsequent invasive cancer diagnoses in the Cox models.

However, this study also had some limitations. Information on potential risk factors such as family history for breast cancer, genetic polymorphisms, parity, and age at first full-term pregnancy that have been associated with invasive cancer risk in previous studies was not available. Additionally, due to the small 
number of subsequent invasive breast cancer cases, we could not investigate separately the risk of ipsilateral vs. contralateral invasive breast cancer. As with most studies focusing on outcomes in cancer patients, the intensive monitoring of patients after their initial BCIS diagnosis, may have increased the probability of detecting the invasive cancer (i.e., selective surveillance bias) in them. Women included in our study might have attended opportunistic mammographic screening for different reasons (e.g., health-consciousness, family history for breast cancer), which could differentiate them from the general population. Unfortunately, the lack of information on the reasons for screening attendance and family history for breast cancer did not allow us to explore this further. Finally, since not all in situ cancer registration is mandatory (i.e., in sites other than the breast), we cannot exclude the possibility that some women in our study might have previously had an in situ cancer of another site that was not registered, and thus, they should have been excluded from our analyses. However, since the most frequent in situ cancers are registered by the cancer registry, a very low number of women might be falsely included in our analyses, if any at all.

In summary, BCIS patients in the canton of Zurich faced approximately 6.9 times higher risk of invasive breast cancer compared to the general population. They additionally faced 1.6 times higher risk of invasive cancer in sites other than the breast. Of all the potential risk factors for a subsequent invasive (breast or non-breast) cancer tested, only age equal to or greater than 70 years old at BCIS diagnosis was statistically significantly associated with a subsequent invasive non-breast cancer diagnosis. Our results support the increased risk for subsequent cancers in BCIS patients reported in the literature. Future studies should establish the risk factors for subsequent cancers, highlight the need for intensive monitoring in this population, and help distinguish BCIS patients who could benefit from systemic therapy to prevent distant cancers.

\section{REFERENCES}

1. Ward EM, DeSantis CE, Lin CC, Kramer JL, Jemal A, Kohler B, et al. Cancer statistics: Breast cancer in situ. CA Cancer J Clin (2015) 65(6):481-95. doi: $10.3322 /$ caac. 21321

2. Karavasiloglou N, Matthes KL, Berlin C, Limam M, Wanner M, Korol D, et al. Increasing trends in in situ breast cancer incidence in a region with no population-based mammographic screening program: results from Zurich, Switzerland 2003-2014. J Cancer Res Clin Oncol (2019) 145(3):653-60. doi: 10.1007/s00432-018-2822-2

3. Levi F, Te VC, Randimbison L, La Vecchia C. Trends of in situ carcinoma of the breast in Vaud, Switzerland. Eur J Cancer (1997) 33(6):903-6. doi: 10.1016/S0959-8049(97)00048-8

4. Bordoni A, Probst-Hensch NM, Mazzucchelli L, Spitale A. Assessment of breast cancer opportunistic screening by clinical-pathological indicators: a population-based study. Br J Cancer (2009) 101(11):1925-31. doi: 10.1038/ sj.bjc. 6605378

5. Bleyer A, Welch HG. Effect of three decades of screening mammography on breast-cancer incidence. N Engl J Med (2012) 367(21):1998-2005. doi: 10.1056/NEJMoa1206809

6. Leonard GD, Swain SM. Ductal carcinoma in situ, complexities and challenges. J Natl Cancer Inst (2004) 96(12):906-20. doi: 10.1093/jnci/djh164

7. Franceschi S, Levi F, La Vecchia C, Randimbison L, Te VC. Second cancers following in situ carcinoma of the breast. Int J Cancer (1998) 77(3):392-5. doi: 10.1002/(SICI)1097-0215(19980729)77:3<392::AID-IJC14>3.0.CO;2-A

\section{DATA AVAILABILITY STATEMENT}

The datasets generated during and/or analyzed during the current study are not publicly available, but are available from the corresponding author upon reasonable request.

\section{ETHICS STATEMENT}

Ethical review and approval were not required for the study on human participants in accordance with the local legislation and institutional requirements. Written informed consent for participation was not required for this study in accordance with the national legislation and the institutional requirements.

\section{AUTHOR CONTRIBUTIONS}

Conception and design: NK and SR. Data acquisition: ML, DK, and MW. Analyzing the data: NK, KM, and GP. Interpretation of the data: NK and SR. Drafting the manuscript: NK. Critically revising the manuscript: NK, KM, GP, ML, DK, MW, and SR. All authors contributed to the article and approved the submitted version.

\section{FUNDING}

This work was supported by Krebsforschung Schweiz under Grant KFS-4114-02-2017 to SR. The funder had no role in study design, data collection and analysis, decision to publish, or preparation of the manuscript.

8. Levi F, Randimbison L, Te VC, La Vecchia C. Invasive breast cancer following ductal and lobular carcinoma in situ of the breast. Int J Cancer (2005) 116 (5):820-3. doi: 10.1002/ijc.20870

9. Sackey H, Hui M, Czene K, Verkooijen H, Edgren G, Frisell J, et al The impact of in situ breast cancer and family history on risk of subsequent breast cancer events and mortality - a population-based study from Sweden. Breast Cancer Res (2016) 18(1):105. doi: 10.1186/ s13058-016-0764-7

10. Soerjomataram I, Louwman WJ, van der Sangen MJ, Roumen RM, Coebergh JW. Increased risk of second malignancies after in situ breast carcinoma in a population-based registry. $\mathrm{Br} J$ Cancer (2006) 95(3):393-7. doi: 10.1038/ sj.bjc. 6603231

11. Falk RS, Hofvind S, Skaane P, Haldorsen T. Second events following ductal carcinoma in situ of the breast: a register-based cohort study. Breast Cancer Res Treat (2011) 129(3):929-38. doi: 10.1007/s10549-011-1531-1

12. Rawal R, Lorenzo Bermejo J, Hemminki K. Risk of subsequent invasive breast carcinoma after in situ breast carcinoma in a population covered by national mammographic screening. Br J Cancer (2005) 92(1):162-6. doi: 10.1038/ sj.bjc. 6602250

13. Eichholzer M, Richard A, Rohrmann S, Schmid SM, Leo C, Huang DJ, et al. Breast cancer screening attendance in two Swiss regions dominated by opportunistic or organized screening. BMC Health Serv Res (2016) 16 (1):519. doi: 10.1186/s12913-016-1760-4

14. Ulyte A, Wei W, Dressel H, Gruebner O, von Wyl V, Bähler C, et al. Variation of colorectal, breast and prostate cancer screening activity in Switzerland: 
Influence of insurance, policy and guidelines. PloS One (2020) 15(4):e0231409. doi: 10.1371/journal.pone.0231409

15. Wanner M, Matthes KL, Korol D, Dehler S, Rohrmann S. Indicators of Data Quality at the Cancer Registry Zurich and Zug in Switzerland. BioMed Res Int (2018) 2018:7656197. doi: 10.1155/2018/7656197

16. Miettinen J, Rantanen M. popEpi: Functions for Epidemiological Analysis using Population Data. R package version 0.4.5. (2018). Available at: https://CRAN. R-project.org/package=popEpi.

17. Therneau TM. A Package for Survival Analysis in R. version 2.38. (2015). Available at: https://CRAN.R-project.org/package=survival.

18. Warnberg F, Yuen J, Holmberg L. Risk of subsequent invasive breast cancer after breast carcinoma in situ. Lancet (2000) 355(9205):724-5. doi: 10.1016/ S0140-6736(99)03703-4

19. Allred DC, Wu Y, Mao S, Nagtegaal ID, Lee S, Perou CM, et al. Ductal carcinoma in situ and the emergence of diversity during breast cancer evolution. Clin Cancer Res (2008) 14(2):370-8. doi: 10.1158/1078-0432.CCR-07-1127
20. Li CI, Malone KE, Saltzman BS, Daling JR. Risk of invasive breast carcinoma among women diagnosed with ductal carcinoma in situ and lobular carcinoma in situ, 1988-2001. Cancer (2006) 106(10):2104-12. doi: 10.1002/ cncr.21864

Conflict of Interest: The authors declare that the research was conducted in the absence of any commercial or financial relationships that could be construed as a potential conflict of interest.

Copyright (c) 2021 Karavasiloglou, Matthes, Pestoni, Limam, Korol, Wanner and Rohrmann. This is an open-access article distributed under the terms of the Creative Commons Attribution License (CC BY). The use, distribution or reproduction in other forums is permitted, provided the original author(s) and the copyright owner(s) are credited and that the original publication in this journal is cited, in accordance with accepted academic practice. No use, distribution or reproduction is permitted which does not comply with these terms. 